\title{
Estudo da capacidade de regularização de vazão na bacia do Ribeirão São Bartolomeu visando abastecimento público
}

\author{
Greicelene Jesus da SILVA ${ }^{*}$, Micael de Souza FRAGA², Demetrius David da SILVA
}

\author{
1Departamento de Engenharia Agrícola, Universidade Federal de Viçosa, Viçosa, MG, Brasil. (ORCID: *; 0000-0001-9666-7421) \\ 2Instituto Mineiro de Gestão das Águas, Belo Horizonte, MG, Brasil. (ORCID: 0000-0002-1996-9343) \\ *E-mail: greicelene.dasilva@gmail.com (ORCID: 0000-0002-2000-4629)
}

Recebido em: 22/04/2019; Aceito em: 01/10/2019; Publicado em: 04/02/2020.

\begin{abstract}
RESUMO: O objetivo do presente trabalho foi avaliar a viabilidade de implantação de novos reservatórios de regularização de vazões na bacia do ribeirão São Bartolomeu, Minas Gerais, Brasil. Foram estudadas duas regiões para implementação de novos reservatórios. O cálculo da capacidade máxima de regularização foi realizado com base na metodologia conhecida como diagrama de massas. Para o dimensionamento da estrutura de emergência, foi realizado o cálculo da vazão máxima de projeto a partir do método racional modificado. A vazão média evaporada do período de estudo resultou em 4,5 $\mathrm{L} \mathrm{s}^{-1}$ e $1,0 \mathrm{~L} \mathrm{~s}^{-1}$ para os reservatórios. Foram obtidos volumes úteis de $375.216 \mathrm{~m}^{3}$ e $66.596 \mathrm{~m}^{3}$ e profundidades máximas de $16,2 \mathrm{~m}$ e $17,7 \mathrm{~m}$. As áreas dos espelhos de água equivaleram a $93.348 \mathrm{~m}^{2}$ e $18.041 \mathrm{~m}^{2}$. Tendo em vista o quadro de escassez hídrica no município de Viçosa, recomenda-se a implantação de um dos reservatórios, com aumento da disponibilidade hídrica para fins de abastecimento público. Entretanto, são necessários maiores estudos em relação à potencialidade de aumento dos conflitos por recursos hídricos na bacia. Adicionalmente, ressalta-se a necessidade da avaliação de potenciais alterações na qualidade da água do rio a jusante dos reservatórios propostos.
\end{abstract}

Palavras-chave: disponibilidade hídrica; ponto de captação; reservatórios.

\section{Study of flow regulation capacity in the São Bartolomeu River basin for water supply}

\begin{abstract}
This paper aimed to evaluate the viability of the implementation of new reservoirs to regulate flow in the São Bartolomeu river basin, Minas Gerais, Brazil. Two regions were studied. The maximum regularization capacity was calculated based on the methodology known as mass diagram. For the dimensioning of the emergency structure, the maximum design flow was calculated from the modified rational method. The mean evaporated flow of the study period resulted in $4.5 \mathrm{~L} \mathrm{~s}^{-1}$ and $1.0 \mathrm{~L} \mathrm{~s}^{-1}$. Useful volumes of $375,216 \mathrm{~m}^{3}$ and $66,596 \mathrm{~m}^{3}$ and maximum depths of $16.2 \mathrm{~m}$ and $17.7 \mathrm{~m}$ were obtained. The water mirrors areas obtained for the new dams amounted to $93,348 \mathrm{~m}^{2}$ and $18,041 \mathrm{~m}^{2}$. Considering the situation of water scarcity in the municipality of Viçosa, it is recommended to install one of the proposed reservoirs, with increased availability of water for public supply. However, further studies are needed regarding the potential for increasing conflicts over water resources in the basin. In addition, the need to evaluate changes in river water quality downstream the proposed reservoirs is emphasized.
\end{abstract}

Keywords: water availability; catchment point; reservoirs.

\section{INTRODUÇÃO}

Diversas regiões do mundo enfrentam severos problemas de falta de água (SHAMSUZZOHA et al., 2017). Cada localidade tem suas próprias adversidades relacionadas à qualidade e quantidade de água em razão do seu clima, geografia, geologia e condições socioeconômicas (PAUL; ELANGO, 2018). Adicionalmente, o crescimento populacional e as mudanças climáticas são vistos como agentes agravantes nesse processo de escassez hídrica (GARCIA-CUERVA et al., 2016).

Escassez hídrica pode ser definida como a exploração excessiva dos recursos hídricos ao ponto de que a demanda se torne superior à disponibilidade (LOON; LANEN, 2013). A escassez hídrica gerada pelo aumento da procura por água, aliada a oferta limitada, representa um dos atuais desafios da gestão de recursos hídricos (LEROUX et al., 2018).
Nesse contexto, a implantação de reservatórios é um eficiente meio de solucionar problemas relacionados à escassez hídrica de forma temporária. Estima-se um gasto mundial de 2 trilhões de dólares em represamentos nas décadas recentes (PEARCE, 2017). Hogeboom et al. (2018) calcularam benefícios econômicos anuais da ordem de 300 bilhões de dólares a partir da produção de 2.235 reservatórios, majoritariamente devido ao valor agregado proveniente de barramentos para geração de energia elétrica e abastecimento residencial e industrial.

Até certo ponto, a escassez hídrica pode ser reduzida a partir do armazenamento de água proveniente do escoamento superficial em reservatórios artificiais no período chuvoso para melhor disponibilidade no seco (ZHUO et al., 2019). Todavia, o represamento de cursos d'água conduz a um grande montante de água evaporada pela superfície 
(ZHAO; LIU, 2015). As perdas por evaporação provenientes de diversos reservatórios, selecionados no estudo de Mekonen; Hoekstra (2012), correspondem anualmente a $10 \%$ do montante de água gastos em produção de culturas. Dessa forma, é essencial o entendimento da dinâmica, especialmente em se tratando das perdas, visando o correto gerenciamento dos reservatórios (ALTHOFF et al., 2019).

Em relação à região de estudo, o abastecimento de parte do município de Viçosa, Minas Gerais, e do campus da Universidade Federal de Viçosa (UFV) é realizado pela captação de água do ribeirão São Bartolomeu, oriunda de regularização proporcionada por barramentos construídos no referido campus.

O desenvolvimento de Viçosa possui conexão histórica com o a implantação e expansão da UFV. Na década de oitenta, o município sofreu crescimento acelerado de $50 \%$ de sua população em comparação à década anterior, majoritariamente devido à ampliação experimentada pela UFV no mesmo período (PEREIRA, 2005). Atualmente, Viçosa ainda apresenta taxas relevantes de acréscimo populacional, uma vez que, entre 2000 e 2010, o número de habitantes cresceu 11\% (IBGE, 2019).

Aliada ao aumento populacional e às mudanças no padrão de consumo experimentado pela população do município, está a ampliação da demanda por recursos hídricos, que apresentou crescimento aproximado de $540.000 \mathrm{~m}^{3}$ entre 2000 e 2007 (RODRIGUES et al., 2009). Dessa forma, a procura crescente de água, associada ao caráter irregular da distribuição espacial e temporal dos recursos hídricos na região, vem afetando a quantidade do recurso disponível para o abastecimento público. A degradação da bacia em estudo tem preocupado diversas autoridades, como o órgão responsável pelo abastecimento e saneamento do município, o Serviço autônomo de Água e Esgoto (SAAE), que relatou uma produção hídrica de $200 \mathrm{~L} \mathrm{~s}^{-1}$ em épocas de estiagem há aproximadamente 40 anos (RODRIGUES et al., 2009) em comparação a médias próximas a $28 \mathrm{~L} \mathrm{~s}^{-1}$ para o período de seca em 2017.

Sendo assim, é imprescindível uma melhoria na gestão dos recursos hídricos na bacia do São Bartolomeu, que pode ser obtida, como medida emergencial, a partir da construção de novos reservatórios de regularização de vazões.

Diante dessa problemática, esse trabalho teve por objetivo verificar a viabilidade de implantação de novos reservatórios de regularização de vazões na bacia do ribeirão São Bartolomeu para fins de abastecimento público do município de Viçosa e da UFV.

\section{MATERIAL E MÉTODOS}

\subsection{Caracterização da área de estudo}

A bacia do ribeirão São Bartolomeu encontra-se inserida na bacia do rio Piranga, com área aproximada de $55,1 \mathrm{Km}^{2}$ (Figura 1), entre as coordenadas geográficas $42^{\circ} 50^{\prime} 56^{\prime \prime} \mathrm{W}$ e $42^{\circ} 52^{\prime} 58^{\prime \prime}$ W de longitude e $20^{\circ} 43^{\prime} 42^{\prime \prime}$ S e $20^{\circ} 50^{\prime} 12^{\prime \prime} \mathrm{S}$ de latitude. A bacia encontra-se totalmente inserida no município de Viçosa (Minas Gerais), o qual possui uma área de aproximadamente 299,4 $\mathrm{Km}^{2}$ e população de 72.220 habitantes (IBGE, 2019).

Em Viçosa, o abastecimento da quase totalidade do município é realizado por duas estações de tratamento de água (ETA), os sistemas ETA I e ETA II, operados pelo
SAAE. A ETA I é abastecida pelo ribeirão São Bartolomeu e o ponto de captação de água está localizado em um dos cinco barramentos localizados no campus da UFV. Já a ETA II é suprida pela captação de água do rio Turvo Sujo.

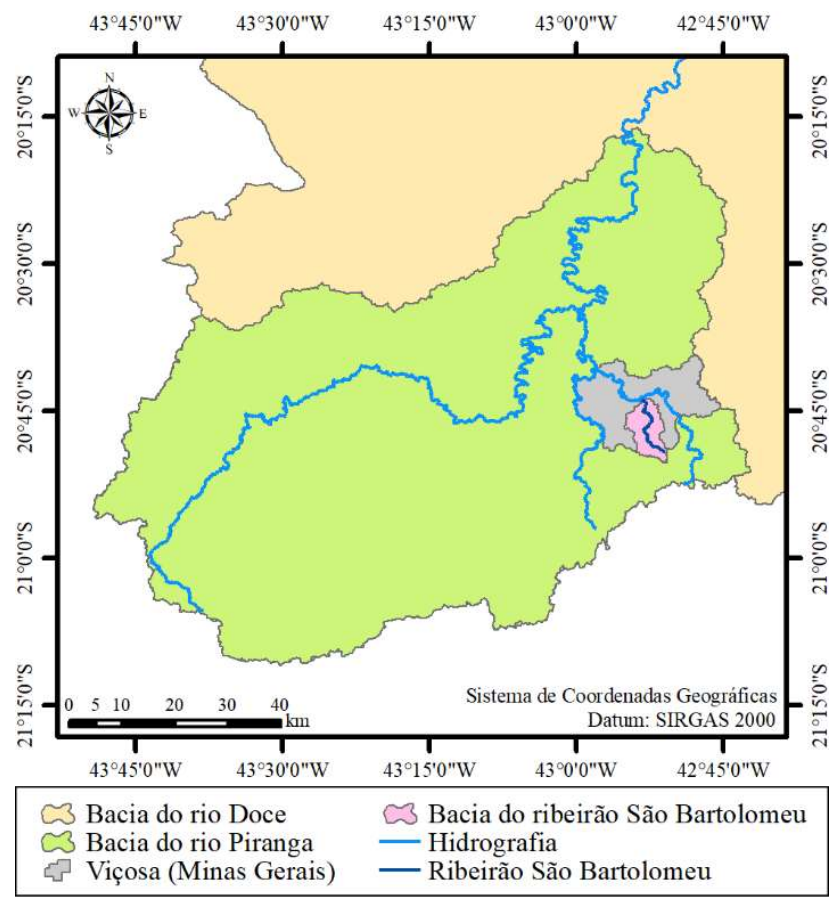

Figura 1. Localização da bacia do ribeirão São Bartolomeu.

Figure 1. Location of the São Bartolomeu river basin.

O ribeirão São Bartolomeu possui cinco barramentos em sequência localizados dentro do campus da UFV, sendo que os dois primeiros (Barramentos 1 e 2) possuem função de regularização de vazão para fins de abastecimento público. A UFV possui suas próprias estruturas de captação, tratamento e abastecimento de água, das quais atendem cerca de 20.000 estudantes e servidores.

\subsection{Regiões propostas para a construção dos novos reservatórios}

Foram avaliados dois locais para a implantação de novos reservatórios no ribeirão São Bartolomeu, ambos a montante das barragens de captação localizadas na UFV. Com base em observações de campo, as regiões propostas atualmente são majoritariamente ocupadas por árvores e vegetação rasteira, havendo poucas infraestruturas públicas ou privadas.

O reservatório proposto identificado como R1 possui uma área de drenagem total de $21,8 \mathrm{~km}^{2}$. Já o reservatório denominado R2 possui uma bacia de contribuição de $2,8 \mathrm{~km}^{2}$ que está contida na bacia de drenagem do R1. Na Figura 2 pode ser visualizada a contextualização espacial das bacias de contribuição dos reservatórios propostos em relação às lagoas já existentes.

\subsection{Batimetria dos barramentos já existentes na UFV}

Os dados de mapeamento do relevo submerso das duas primeiras lagoas localizadas no campus da UFV, que foram utilizados para o cálculo do volume útil real dos reservatórios existentes, provêm de estudos realizados por Carmo; Ferreira (2012). Nesse estudo foram obtidos os volumes entre a lâmina d'água e a superfície batimétrica, expressos na Tabela 1. 
Tabela 1. Valores de perímetro, área e volume dos barramentos localizados no campus da UFV.

Table 1. Perimeter, area and volume of the reservoirs located on the UFV campus.

\begin{tabular}{cccc}
\hline Barramento & Perímetro $(\mathrm{m})$ & Área (ha) & Vol. $\left(\mathrm{m}^{3}\right)$ \\
\hline 1 & 1.511 & 3,12 & 62.050 \\
2 & 1.715 & 3,20 & 81.220 \\
\hline
\end{tabular}

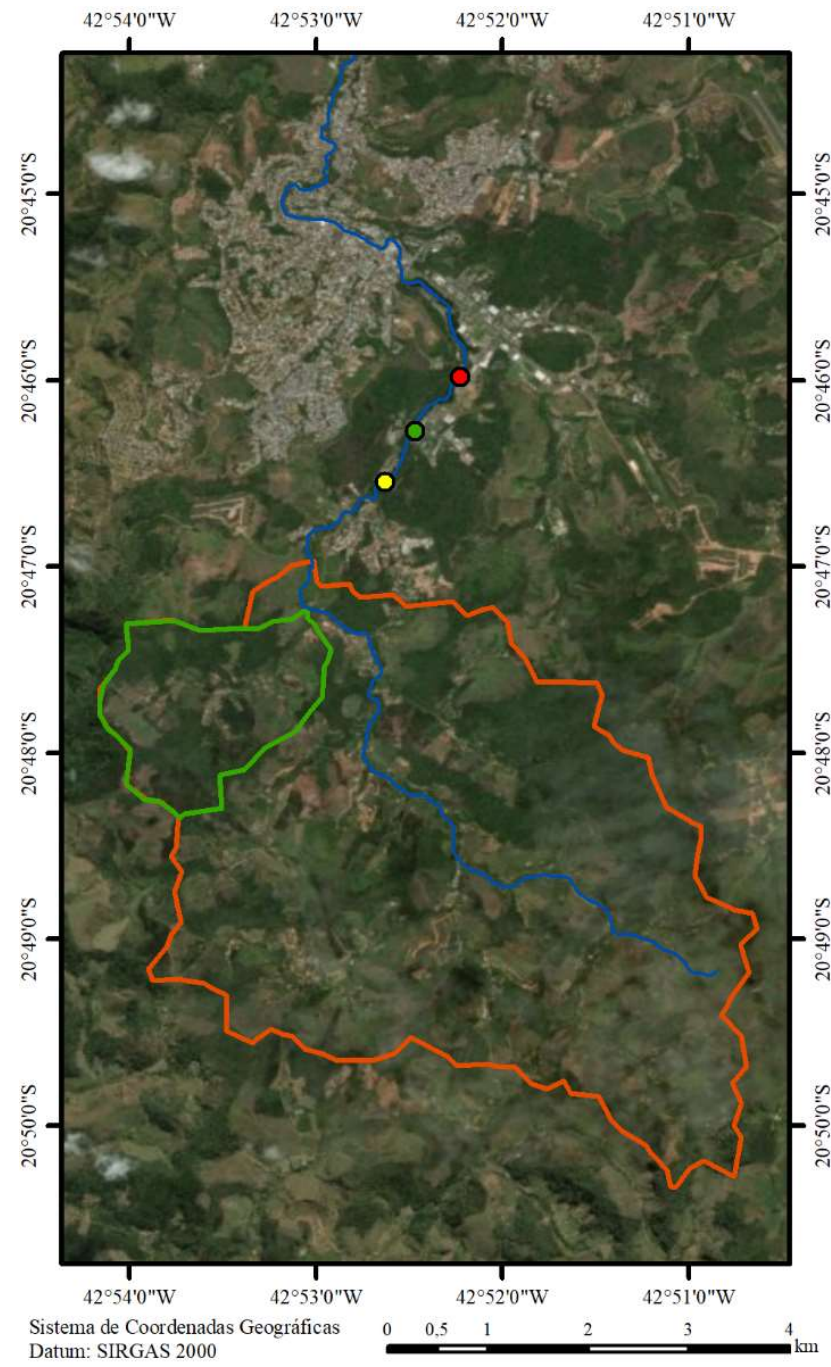

\begin{tabular}{|c|c|c|}
\hline & $\begin{array}{l}\text { - Ribeirão São Bartolomeu } \\
\text { ב Área de contribuição do R1 } \\
\mathbb{J} \text { Área de contribuição do R2 }\end{array}$ & $\begin{array}{ll}\text { O } & \text { Ponto de medição } \\
\text { O } & \text { Barramento } 1 \\
\text { B } & \text { Barramento } 2\end{array}$ \\
\hline
\end{tabular}

Figura 2. Contextualização espacial das áreas de contribuição dos reservatórios propostos.

Figure 2. Spatial contextualization of the proposed reservoirs contribution areas.

\subsection{Dimensionamento da capacidade de acumulação dos reservatórios propostos}

A metodologia empregada para o cálculo do volume útil do reservatório utilizada no presente estudo foi a proposta por Rippl (1883), também conhecida como diagrama de massas. Os dados de vazão do ribeirão São Bartolomeu provieram de monitoramentos rotineiros efetuados diariamente pela Divisão de Água e Esgoto da UFV. A medida é constantemente realizada a partir do emprego do molinete hidráulico a montante do barramento 1 (Figura 2), de coordenadas $42^{\circ} 52^{\prime} 43^{\prime \prime} \mathrm{W}$ e $20^{\circ} 46^{\prime} 38^{\prime \prime} \mathrm{S}$, correspondente a uma área de contribuição de $25 \mathrm{Km}^{2}$.

Para fins do estudo, avaliou-se a capacidade máxima de regularização, obtida a partir vazão média para o período de novembro de 2014 a outubro de 2017, descontando-se as perdas por evaporação. A vazão específica calculada para a bacia na qual é realizada a medição da vazão do ribeirão São Bartolomeu serviu de base para a espacialização dos dados e determinação da produção hídrica mensal das bacias de contribuição estudadas.

$\mathrm{Na}$ determinação do volume útil do reservatório projetado para a bacia maior, identificado no estudo como $\mathrm{R} 1$, além de descontar-se do volume obtido no diagrama de massas as perdas por evaporação, realizou-se também a subtração do montante já armazenado nos barramentos de captação existentes a jusante, conforme obtido por Carmo; Ferreira (2012). Ressalta-se que, por questões de simplificação do estudo, nesse trabalho não foram consideradas as perdas por infiltração em ambos os reservatórios propostos.

\subsection{Estimativa da evaporação e cálculo da vazão equivalente à lâmina evaporada}

Para a estimativa da evaporação nos reservatórios, foram utilizados dados de precipitação, temperatura, umidade relativa, velocidade do vento e insolação, obtidos da estação meteorológica convencional localizada em Viçosa, código

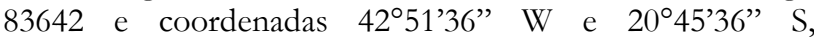
pertencente à rede do Instituto Nacional de Meteorologia (INMET).

Os dados utilizados compreenderam o período de novembro de 2014 a outubro de 2017 . A evaporação também foi obtida a partir das normais climatológicas do período de 1981 a 2010 para Viçosa, disponibilizadas pelo INMET. O procedimento utilizado no cálculo da evaporação nos reservatórios propostos foi o mesmo adotado por Penman $(1948,1956)$. O albedo da água foi considerado equivalente a 0,11 , valor obtido por Giongo; Vettorazzi (2014), adequado a reservatórios brasileiros.

A partir dos valores estimados de lâmina de evaporação na região de estudo, foi possível obter a vazão média evaporada referente aos reservatórios propostos para o período estudado. O cálculo foi realizado a partir da Equação 1.

$$
\mathrm{Q}_{\mathrm{EV}}=\frac{\mathrm{EV} * \mathrm{~A}}{86400} \quad(\mathrm{Eq} .1)
$$

em que: $\mathrm{Q}_{\mathrm{EV}}=$ vazão evaporada no reservatório $\left(\mathrm{m}^{3} \mathrm{~s}^{-1}\right) ; \mathrm{EV}=$ lâmina de evaporação diária do reservatório $\left(\mathrm{m} \mathrm{dia}^{-1}\right) ; \mathrm{A}=$ área do espelho de água dos reservatórios propostos $\left(\mathrm{m}^{2}\right)$.

\subsection{Obtenção do Modelo Digital de Elevação Hidrograficamente Condicionado (MDEHC)}

Com o objetivo de realizar o estudo hidrológico das áreas propostas, foi obtido o Modelo Digital de Elevação Hidrograficamente Condicionado (MDEHC) das áreas de contribuição do R1 e R2, a partir de um MDE de resolução de $5 \mathrm{~m}$, com o auxílio do software ArcGIS/ArcMap $10.1 \mathrm{da}$ ESRI. O MDE proveio de uma imagem Ikonos de $1 \mathrm{~m}$ de resolução. A metodologia utilizada na obtenção do MDEHC foi a mesma descrita por Pereira et al. (2016). 


\subsection{Determinação da área inundada e obtenção da curva Cota $\times$ Volume}

A obtenção do espelho de água dos reservatórios de interesse foi realizada com $\mathrm{O}$ auxílio do software ArcGIS/ArcMap 10.1 da ESRI. A metodologia consistiu na estimativa da área de uma curva de nível de determinada altitude abaixo da qual está contido o volume referente ao calculado conforme o procedimento proposto por Rippl (1883). No caso do R1, o montante refere-se ao volume obtido pela dedução do total armazenado pelas lagoas de captação existentes na UFV.

A partir do MDEHC, foram geradas curvas de nível de 1 $\mathrm{m}$ em $1 \mathrm{~m}$, iniciando da cota mínima indicada no MDEHC até a altitude correspondente à altura máxima da lâmina d'água no vertedor de emergência. Dessa forma, foi calculado o volume acumulado associado a cada curva de nível consecutiva.

\subsection{Estimativa da vazão máxima de projeto}

$\mathrm{Na}$ estimativa do escoamento superficial, foi considerada a cheia máxima de projeto para um período de retorno de 100 anos. O cálculo foi feito com base no método racional modificado (Equação 2). O coeficiente de retardamento foi determinado a partir da equação ajustada obtida no trabalho de Euclydes (1987), na região Sul de Minas Gerais (Equação 3).

$$
\mathrm{Q}_{\text {máx }}=\frac{\mathrm{C} * \mathrm{I}_{\mathrm{m}} * \mathrm{~A}}{360} * \phi
$$

em que: $\mathrm{Q}_{\text {máx }}=$ vazão máxima de escoamento superficial $\left(\mathrm{m}^{3} \mathrm{~s}^{-1}\right) ; \mathrm{C}$ $=$ coeficiente de escoamento superficial (adimensional); $\mathrm{I}_{\mathrm{m}}=$ intensidade máxima média de precipitação para uma duração igual ao tempo de concentração da bacia $\left(\mathrm{mm} \mathrm{h}^{-1}\right) ; \mathrm{A}$ = área da bacia (ha); $\phi=$ coeficiente de retardamento (adimensional).

$$
\phi=0,278-0,00034 * \mathrm{~A}
$$

em que: $\mathrm{A}=\mathrm{a}$ área da bacia $\left(\mathrm{km}^{2}\right)$.

\subsubsection{Determinação do coeficiente de escoamento superficial}

O coeficiente de escoamento superficial (C) representa a relação entre o volume que escoa sobre a superfície do terreno (ES) e o volume total precipitado (PT). Os valores dos coeficientes de escoamento superficial utilizados no presente estudo hidrológico foram obtidos a partir da metodologia proposta por Chow et al. (1988), na qual considera vários tipos de cobertura da superfície, declividade e períodos de retorno.

Como há variação do coeficiente de escoamento superficial ao longo da área analisada, o coeficiente global foi determinado com base na Equação 4.

$$
\mathrm{C}=\frac{\sum_{\mathrm{i}=1}^{\mathrm{n}} \mathrm{C}_{\mathrm{i}} * \mathrm{~A}_{\mathrm{i}}}{\mathrm{A}}
$$

em que: $\mathrm{C}=$ coeficiente de escoamento superficial para a área de interesse (adimensional); $\mathrm{C}_{\mathrm{i}}=$ coeficiente de escoamento superficial para a subárea $\mathrm{i}$ (adimensional); $\mathrm{A}_{\mathrm{i}}=$ subárea considerada (ha); $\mathrm{A}=$ área da bacia (ha).

$\mathrm{Na}$ segregação das diferentes regiões, foi gerado o mapa de declividades diferenciadas em plano (0-2\%), médio (2-7\%) e inclinado $(>7 \%)$. No processo de obtenção do coeficiente
C, foi adotada como base a classificação de uso e ocupação do solo para a bacia do ribeirão São Bartolomeu, segundo trabalho de Pereira et al. (2017). Os mapas de classificação para ambas as bacias propostas são encontrados nas Figuras 3 e 4.

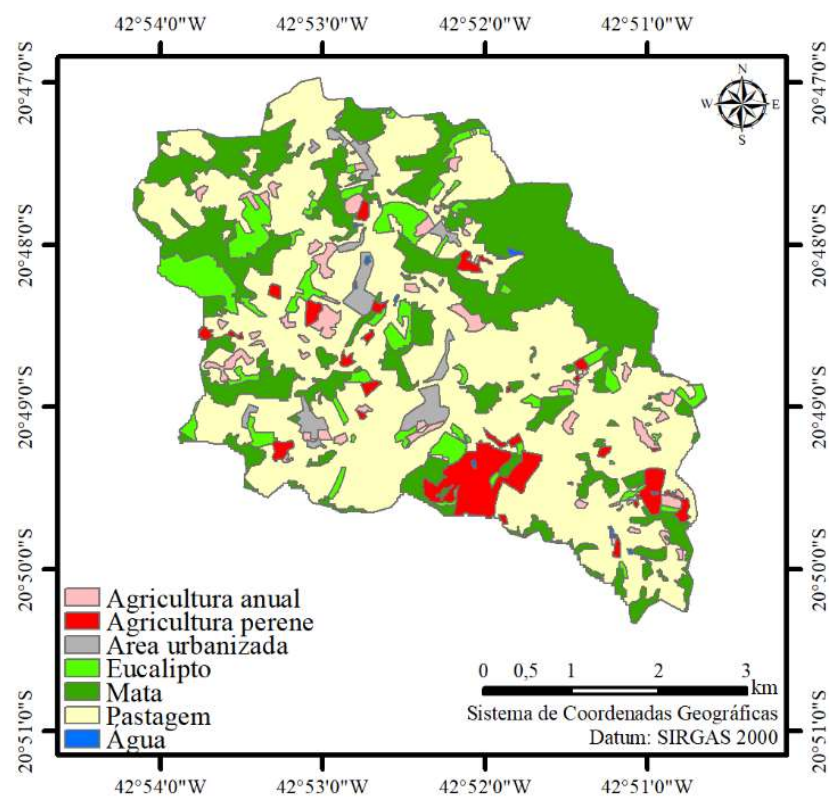

Figura 3. Uso do solo para a bacia de contribuição do R1. Fonte: Pereira et al. (2017).

Figure 3. Soil use for the R1 contribution basin. Source: Pereira et al. (2017).

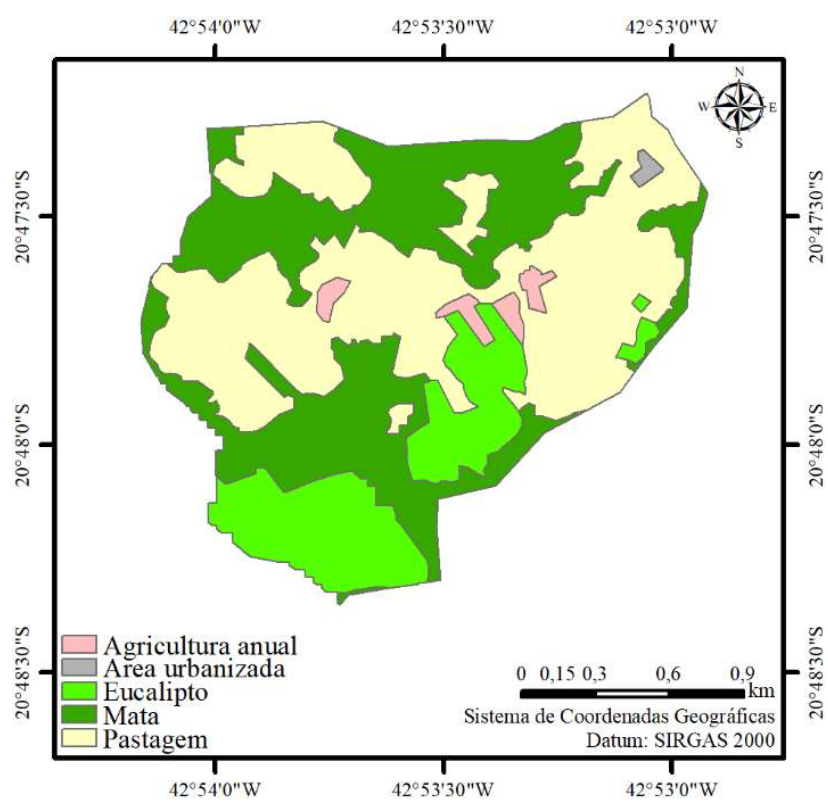

Figura 4. Uso do solo para a bacia de contribuição do R2. Fonte: Pereira et al. (2017).

Figure 4. Soil use for the R2 contribution basin. Source: Pereira et al. (2017).

As distintas classes de uso e ocupação do solo identificadas no trabalho de Pereira et al. (2017) receberam diferentes coeficientes de escoamento superficial, adequando-as à metodologia proposta por Chow et al. (1988). Considerou-se, ainda, a declividade do terreno, adotando-se um período de retorno de 100 anos (Tabela 2). 
Tabela 2. Valores de $\mathrm{C}$ adotados no estudo.

Table 2. $\mathrm{C}$ values adopted in the study.

\begin{tabular}{|c|c|c|c|}
\hline $\begin{array}{c}\text { Tipo de } \\
\text { Cobertura* }\end{array}$ & $\begin{array}{l}\text { Classe de uso } \\
\text { adotada** }\end{array}$ & Declividade & $\begin{array}{c}\mathrm{C} \\
\text { adotado }\end{array}$ \\
\hline \multirow{3}{*}{$\begin{array}{l}\text { Área urbana e } \\
\text { lotes }\end{array}$} & \multirow{3}{*}{$\begin{array}{l}\text { Concreto/ } \\
\text { telhado }\end{array}$} & Plano & 0,97 \\
\hline & & Médio & 0,97 \\
\hline & & Inclinado & 0,97 \\
\hline \multirow{3}{*}{$\begin{array}{l}\text { Agricultura } \\
\text { anual e perene }\end{array}$} & \multirow{3}{*}{$\begin{array}{l}\text { Campos } \\
\text { cultivados }\end{array}$} & Plano & 0,47 \\
\hline & & Médio & 0,51 \\
\hline & & Inclinado & 0,54 \\
\hline \multirow{3}{*}{ Pastagem } & \multirow{3}{*}{ Pastos } & Plano & 0,41 \\
\hline & & Médio & 0,49 \\
\hline & & Inclinado & 0,53 \\
\hline \multirow{3}{*}{$\begin{array}{l}\text { Mata e } \\
\text { eucalipto }\end{array}$} & \multirow{3}{*}{$\begin{array}{c}\text { Floresta/ } \\
\text { reflorestamento }\end{array}$} & Plano & 0,39 \\
\hline & & Médio & 0,47 \\
\hline & & Inclinado & 0,52 \\
\hline
\end{tabular}

*Conforme classificação de Pereira et al. (2017)

**Conforme metodologia de Chow et al. (1988)

\subsubsection{Intensidade máxima média da precipitação}

A intensidade considerada para a aplicação do método racional modificado foi a máxima média $\left(\mathrm{I}_{\mathrm{m}}\right)$ observada para uma duração correspondente ao tempo de concentração $\left(t_{c}\right)$ e para o período de retorno $(\mathrm{T})$ estabelecido no estudo. A equação da intensidade (Equação 5) para a cidade de Viçosa foi obtida por meio do software Pluvio 2.1 - Chuvas Intensas para o Brasil.

$$
I_{m}=\frac{3510,725 * T^{0,227}}{\left(t_{c}+2,319\right)^{0,995}}
$$

em que: $\mathrm{I}_{\mathrm{m}}=$ intensidade máxima média de precipitação $\left(\mathrm{mm} \mathrm{h}^{-1}\right)$; $\mathrm{T}=$ período de retorno $(\operatorname{anos}) ; \mathrm{t}_{\mathrm{c}}=$ duração da precipitação equivalente ao período de retorno, obtida com base na equação de Kirpich (min).

No cálculo da extensão do talvegue, foi obtido, no MDEHC, o comprimento de uma linha reta traçada desde a foz até o ponto mais remoto da região de interesse. Por fim, mensurou-se a declividade do rio principal a partir da diferença de nível entre o ponto mais remoto da bacia e a seção de deságue. Esses pontos equivalem, respectivamente, ao maior e menor valor de elevação aferido no MDEHC.

\subsubsection{Dimensionamento das estruturas de descarga considerando a cheia máxima}

No dimensionamento do vertedor para extravasamento das vazões máximas de enchente foi empregada a equação de um vertedor retangular de parede espessa sem contração (equação de Lesbrós) (MATOS et al. 2012). No presente estudo, a altura da lâmina de água adotada no vertedor de emergência equivaleu a 1,5 m.

\section{RESULTADOS}

3.1. Vazões espacializadas para as regiões de interesse

$\mathrm{Na}$ Tabela 3, encontram-se as vazões espacializadas com base no período de estudos.

Tabela 3. Vazões espacializadas para o período de estudo. Table 3. Spatial stream flows for the study period.

\begin{tabular}{ccc}
\hline $\begin{array}{c}\text { Médias mensais } \\
\text { afluente }\left(\mathrm{L} \mathrm{s}^{-1}\right)\end{array}$ & $\begin{array}{c}\text { Vazão média } \\
\text { espacializada bacia } \\
\text { R1 }\left(\mathrm{L} \mathrm{s}^{-1}\right)\end{array}$ & $\begin{array}{c}\text { Vazão média } \\
\text { espacializada bacia } \\
\text { R2 }\left(\mathrm{L} \mathrm{s}^{-1}\right)\end{array}$ \\
\hline 63,7 & 55,5 & 7,2 \\
\hline
\end{tabular}

\subsection{Elementos meteorológicos e vazão equivalente à} lâmina evaporada

Nas Figuras 5, 6, 7 e 8 encontram-se os valores médios mensais dos elementos meteorológicos precipitação acumulada, temperatura, insolação diária e velocidade do vento para o período de 1981 a 2010 em Viçosa.

Nas Figuras 9 e 10, é apresentada a estimativa da evaporação utilizando a metodologia de Penman (1948, 1956), tomando por base valores diários e mensais dos elementos meteorológicos. A base de dados mensais corresponde às normais climatológicas do período de 1981 a 2010 para a região de Viçosa.

$\mathrm{Na}$ Tabela 4, encontram-se os montantes evaporados anuais. Os dados da vazão média de evaporação, com base nos dados diários de lâmina evaporada e nas áreas do espelho d'agua obtido encontram-se dispostos na Tabela 5.

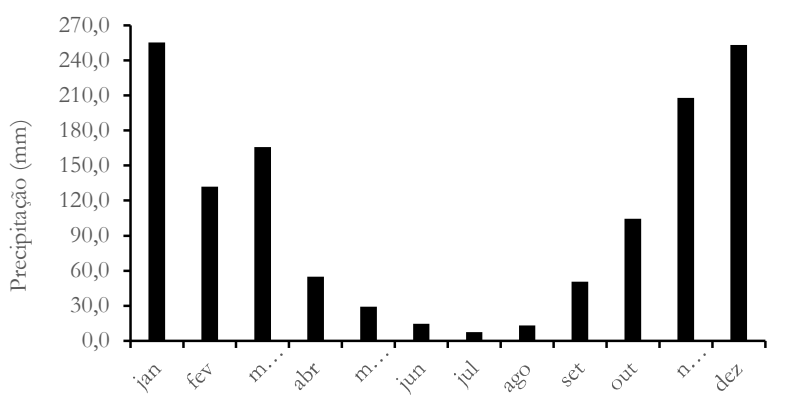

Figura 5. Variação mensal da precipitação para Viçosa, considerando o período de 1981 a 2010.

Figure 5. Monthly variation of precipitation for Vicosa, considering the period from 1981 to 2010 .

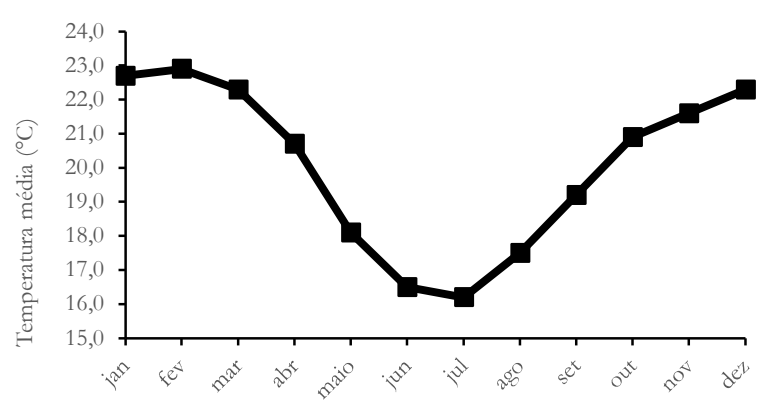

Figura 6. Variação da temperatura média para Viçosa, considerando o período de 1981 a 2010.

Figure 6. Variation of the average temperature for Vicosa, considering the period from 1981 to 2010.

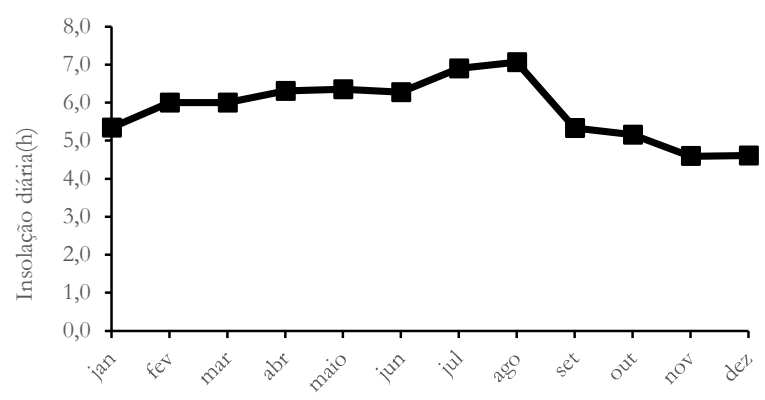

Figura 7. Variação da insolação média para Viçosa, considerando o período de 1981 a 2010.

Figure 7. Variation of average sunshine for Vicosa, considering the period from 1981 to 2010. 
Silva et al.

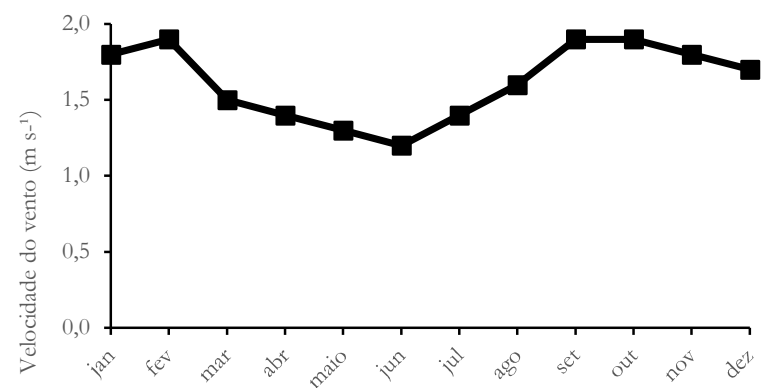

Figura 8. Variação da velocidade do vento média para Viçosa, considerando o período de 1981 a 2010.

Figure 8. Variation of the average wind speed for Viçosa, considering the period from 1981 to 2010 .

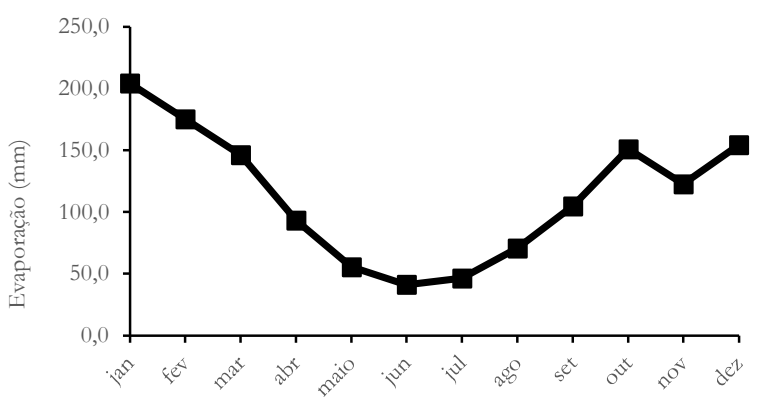

Figura 9. Evaporação mensal com base em dados diários.

Figure 9. Monthly evaporation based on daily data.

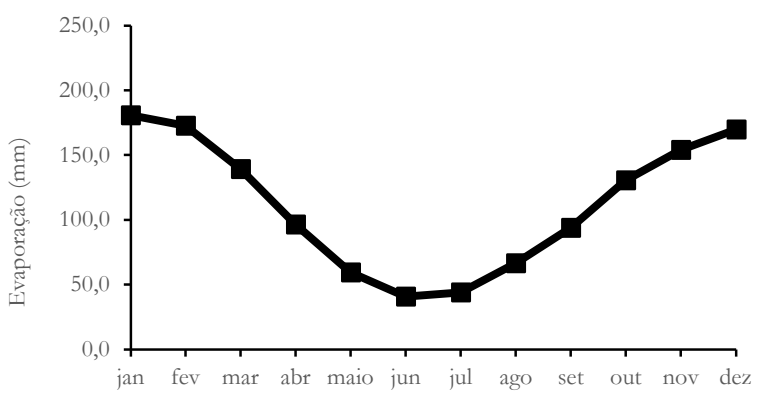

Figura 10. Evaporação mensal com base em normais climatológicas do período de 1981 a 2010.

Figure 10. Monthly evaporation based on climatological normals from 1981 to 2010.

Tabela 4. Evaporação total anual (mm).

Table 4. Annual total evaporation ( $\mathrm{mm}$ ).

\begin{tabular}{lc}
\hline \multicolumn{1}{c}{ Dados } & Total anual \\
\hline Evaporação total com base em dados diários & 1.366 \\
$\begin{array}{l}\text { Evaporação total com base nas normais } \\
\text { climatológicas }\end{array}$ & 1.347 \\
\hline
\end{tabular}

Tabela 5. Vazão média anual evaporada $\left(\mathrm{L} \mathrm{s}^{-1}\right)$.

Table 5. Average annual evaporated flow $\left(\mathrm{L} \mathrm{s}^{-1}\right)$.

\begin{tabular}{cc}
\hline Reservatório & Vazão média evaporada do período de estudo \\
\hline R1 & 4,5 \\
R2 & 1,0 \\
\hline
\end{tabular}

\subsection{Volume útil e dados morfométricos dos reservatórios}

$\mathrm{Na}$ Tabela 6, estão dispostas a capacidade de regularização obtida a partir do diagrama de massas, além do volume útil dos reservatórios, do qual foram descontadas as perdas por evaporação. Os dados morfométricos para ambos os reservatórios propostos encontram-se na Tabela 7. A perspectiva em planta da área de inundação da bacia de contribuição dos reservatórios está apresentada nas Figuras 11 e 12.

Tabela 6. Volume útil dos reservatórios. Table 6. Useful volume of the reservoirs.

\begin{tabular}{ccc}
\hline Reservatório & $\begin{array}{c}\text { Capacidade de } \\
\text { regularização obtida pelo } \\
\text { diagrama de massas }\left(\mathrm{m}^{3}\right)^{*}\end{array}$ & $\begin{array}{c}\text { Volume útil } \\
\left(\mathrm{m}^{3}\right)\end{array}$ \\
\hline R1 & 530.054 & 375.216 \\
R2 & 68.762 & 66.596 \\
\hline
\end{tabular}

*Capacidade de regularização obtida pela subtração do valor já reservado e das perdas por evaporação.

Tabela 7. Dados morfométricos dos reservatórios propostos.

Table 7. Morphometric data of the proposed reservoirs.

\begin{tabular}{|c|c|c|}
\hline Característica & R1 & $\mathrm{R} 2$ \\
\hline Cota do nível normal $\left(\mathrm{H}_{\mathrm{n}}\right)$ & $676,2 \mathrm{~m}$ & $682,7 \mathrm{~m}$ \\
\hline $\begin{array}{l}\text { Cotas do nível máximo no } \\
\text { extravasor }\left(\mathrm{H}_{\mathrm{e}}\right)\end{array}$ & $677,7 \mathrm{~m}$ & $684,2 \mathrm{~m}$ \\
\hline $\begin{array}{l}\text { Profundidade } \\
\text { máxima }\left(\mathrm{D}_{\max }\right)\end{array}$ & $16,2 \mathrm{~m}$ & $17,7 \mathrm{~m}$ \\
\hline Folga (f) & $1,5 \mathrm{~m}$ & $1,5 \mathrm{~m}$ \\
\hline Altura total $(\mathrm{H})$ & $19,2 \mathrm{~m}$ & $20,7 \mathrm{~m}$ \\
\hline Espelho d'água (A) & $96.348 \mathrm{~m}^{2}$ & $18.041 \mathrm{~m}^{2}$ \\
\hline Área da bacia $\left(A_{B}\right)$ & $21,8 \mathrm{Km}^{2}$ & $2,8 \mathrm{Km}^{2}$ \\
\hline $\begin{array}{l}\text { Profundidade } \\
\text { relativa }\left(\mathrm{D}_{\text {rel }}\right)\end{array}$ & $4,6 \%$ & $11,7 \%$ \\
\hline $\begin{array}{l}\text { Relação área da bacia / área do } \\
\text { reservatório }\end{array}$ & 226 & 157 \\
\hline
\end{tabular}

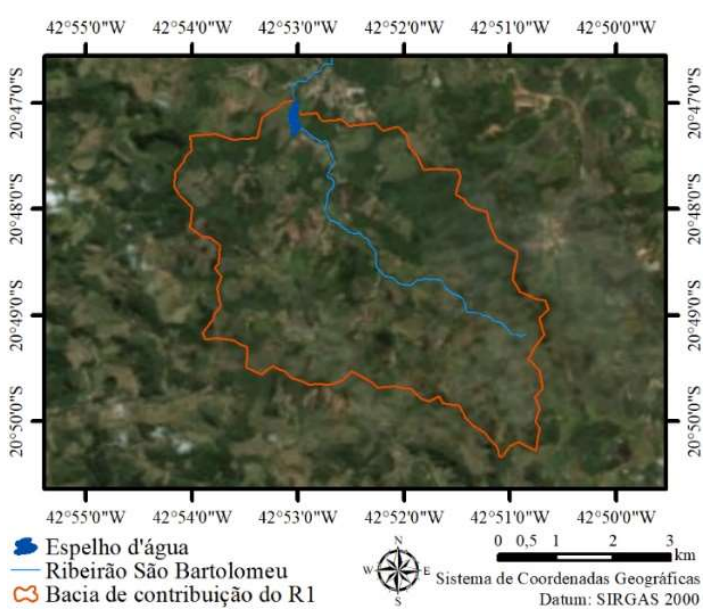

Figure 11. Water mirror for reservoir R1.

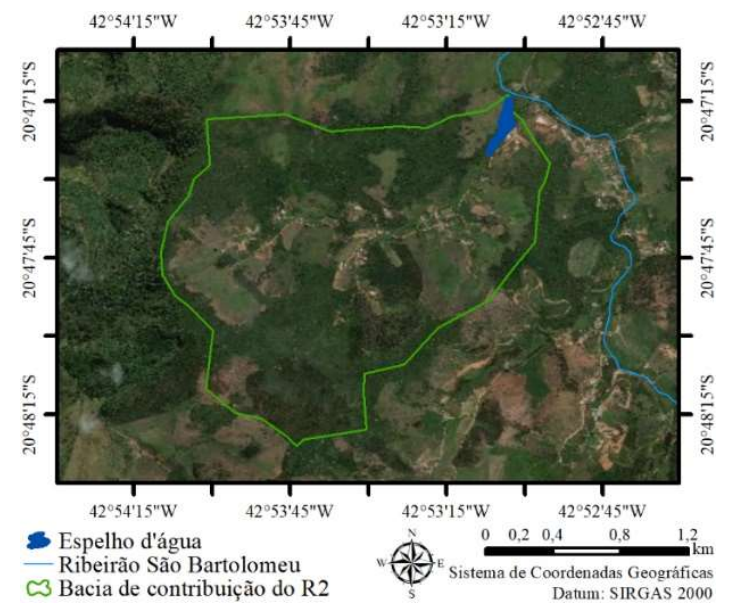

Figura 12. Espelho d'água formado pelo R2.

Figure 12. Water mirror for reservoir R2.

Nativa, Sinop, v. 8, n. 1, p. 102-111, jan./fev. 2020. 


\subsection{Curva Cota $\times$ Volume dos reservatórios propostos}

As curvas de volume total em função da cota do nível de água encontram-se disposta nos gráficos das Figuras 13 e 14 para os reservatórios R1 e R2.

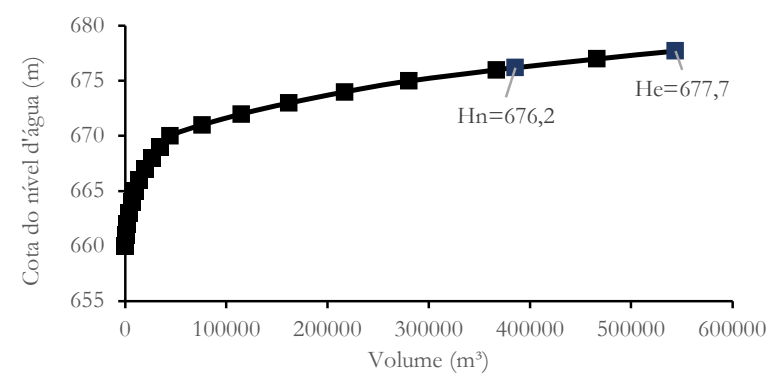

Figura 13. Curva cota $\times$ volume do R1.

Figure 13. Water level $\times$ volume of reservoir R1.

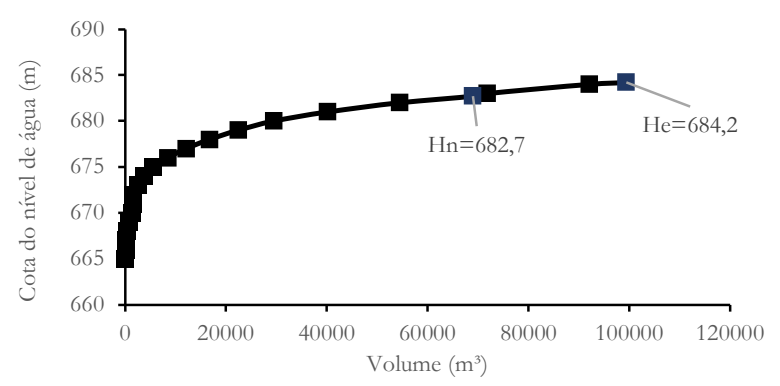

Figura 14. Curva cota $\times$ volume do R2.

Figure 14. Water level $\times$ volume of reservoir $R$.

\subsection{Estimativa da vazão máxima de projeto e largura do vertedor de emergência}

$\mathrm{Na}$ Tabela 8 estão dispostos os valores do coeficiente global de escoamento superficial para ambas as bacias de interesse, bem como os valores dos parâmetros utilizados para o cálculo da intensidade máxima média de precipitação $\left(\mathrm{I}_{\mathrm{m}}\right)$ e a vazão máxima de projeto $\left(\mathrm{Q}_{\operatorname{máx}} \mathrm{T}=100\right)$ para o período de retorno de 100 anos.

Tabela 8. Valores de vazão máxima e parâmetros da equação de chuvas intensas.

Table 8. Maximum flow values and parameters of the intense rainfall equation.

\begin{tabular}{cccccc}
\hline Bacia & $\mathrm{C}$ & $\begin{array}{c}\mathrm{t}_{\mathrm{c}} \\
(\mathrm{min})\end{array}$ & $\begin{array}{c}\mathrm{I}_{\mathrm{m}} \\
\left(\mathrm{mm} \mathrm{h}^{-1}\right)\end{array}$ & $\phi$ & $\begin{array}{c}\mathrm{Q}_{\text {máx } \mathrm{T}=100} \\
\left(\mathrm{~m}^{3} \mathrm{~s}^{-1}\right)\end{array}$ \\
\hline 1 & 0,53 & 73 & 99 & 0,27 & 86 \\
2 & 0,51 & 19 & 212 & 0,28 & 24 \\
\hline
\end{tabular}

Encontram-se na Tabela 9, os valores da largura do vertedor recomendado para o extravasor de emergência dimensionado a partir das vazões máximas.

Tabela 9. Largura do vertedor para extravasamento da vazão máxima de enchente.

Table 9. Width of pourer for extravasation of maximum flood flow.

\begin{tabular}{cc}
\hline Reservatório & Largura recomendada do vertedor $(\mathrm{m})$ \\
\hline $\mathrm{R} 1$ & 11,1 \\
$\mathrm{R} 2$ & 4,7 \\
\hline
\end{tabular}

\section{DISCUSSÃO}

\subsection{Elementos meteorológicos e vazão equivalente à lâmina evaporada}

Em relação ao comportamento da precipitação (Figura 5), pode ser observado que as maiores lâminas precipitadas ocorrem principalmente nos meses de novembro, dezembro e janeiro. Assim, novembro representa o início do ano hidrológico na região.

É evidenciado, nos gráficos das Figuras 9 e 10, um progressivo aumento da evaporação mensal a partir do mês de junho. A curva atinge um ápice em janeiro, a partir do qual os índices se tornam decrescentes, chegando a valores mínimos nos meses de maio, junho e julho. Nesses meses são observados baixos valores de temperatura, precipitação e velocidade do vento (Figuras 5, 6, e 8). Durante esse período, são registrados altos índices de insolação (Figura 9) devido à baixa interferência provocada pelas nuvens na medição.

Nota-se, pela análise do comportamento do gráfico da Figura 9 em comparação ao da Figura 10, obtido a partir de médias de dados mensais, que a evaporação calculada com base nas normais reduz os valores extremos, amortecendo os picos. Entretanto, as lâminas evaporadas anuais, encontradas para ambos os procedimentos (Tabela 4), apresentam valores próximos.

A vazão evaporada nos reservatórios $\mathrm{R} 1$ e $\mathrm{R} 2$ representa, respectivamente, $10,4 \%$ e $1,7 \%$ da vazão natural média da bacia onde é feito o monitoramento da vazão do ribeirão São Bartolomeu, além de $11,9 \%$ e $15,3 \%$ da vazão média mensal proveniente das séries obtidas por espacialização para os reservatórios $\mathrm{R} 1$ e $\mathrm{R} 2$, respectivamente.

\subsection{Curva Cota $\times$ Volume dos reservatórios}

Observa-se na Figura 13 a distribuição desigual do montante armazenado devido às características declivosa do terreno, uma vez que, considerando o volume armazenado abaixo do nível normal, quase metade do montante total armazenado se encontra acima da cota 674 , ou seja, em até 2,2 $\mathrm{m}$ de profundidade. Situação similar pode ser notada para o reservatório R2, como mostra a Figura 14, na qual cerca de $50 \%$ do montante seria mantido acima da cota 681 (até $1,7 \mathrm{~m}$ de profundidade).

\subsection{Viabilidade de implantação dos novos reservatórios na região de estudo}

$\mathrm{O}$ ajuste de uma vazão mínima constante produz alterações na magnitude, frequência, duração, sazonalidade e periodicidade das vazões naturais do rio, o que provoca uma série de mudanças na dinâmica fluvial e nos ecossistemas aquáticos (TORRES et al., 2015). Dessa forma, a regularização do regime hídrico pode trazer efeitos positivos e negativos. Dentre as consequências proveitosas da implantação dos reservatórios na região em estudo, pode-se citar o aumento da disponibilidade hídrica para captação destinada ao abastecimento público, além da diminuição das vazões máximas e consequente atenuação dos transtornos relacionados a inundações.

No entanto, com a regularização do regime de vazões, aumenta-se o potencial de ocorrência de condições específicas de conflito de uso entre as demandas existentes a nível de bacia hidrográfica (MARTINS et al., 2011). Torres et al., (2015), avaliando os efeitos da regularização do Rio São Francisco, analisou vários cenários caracterizados por acentuado conflito de recursos hídricos entre diversos usos, 
destinados à geração de energia, pesca, agricultura de subsistência e manutenção do ecossistema aquático. Objetivando estudar impactos socioambientais provenientes da implantação da usina hidrelétrica de Sobradinho, o trabalho de Martins et al. (2011) identificou que os solos de várzea aproveitados periodicamente pelos ribeirinhos alcançaram reduzidos níveis de nutrientes em virtude da não ocorrência das grandes cheias. De acordo com a análise realizada por Holanda et al. (2009) baseada em relatos da população ribeirinha do Baixo São Francisco sergipano, a construção dos complexos de Sobradinho e Xingó modificou as características de qualidade da água do rio, adversas à reprodução de peixes, o que potencializou a diminuição e até mesmo extinção de espécies.

O estudo da morfologia é fundamental na compreensão dos processos que influenciam no transporte de nutrientes $\mathrm{e}$ na distribuição das comunidades biológicas (KOLADA, 2014). Dentre parâmetros limnológicos comuns, destaca-se a profundidade relativa. Baixos índices de profundidade relativa indicam ótima capacidade de circulação completa ao longo do ano, a qual proporciona oxigenação do sedimento e saturação da massa líquida (SCHÄFER et al., 2014). Os valores da profundidade relativa para as represas propostas (Tabela 7) são consideravelmente elevados tomando por base os estudos de Schäfer et al. (2014), que obteve valores variando de $0,05 \%$ a $0,7 \%$ para 18 lagoas localizadas no Rio Grande do Sul. Isso se deve à topografia declivosa da região do presente estudo, conforme exposto no item 4.2. Neto; Coelho (2002) analisando a relação entre a morfometria e o estado trófico da lagoa do Nado em Belo Horizonte, Minas Gerais, identificaram uma profundidade relativa correspondente a 5,5\%, tais valores sugerem alta estabilidade e estratificação térmica, além de problemas de aeração na massa líquida e geração de extensas zonas anóxica (MORENO et al., 2011), fatores que contribuíram para a maior incidência de condições tipicamente eutróficas em determinados períodos do ano.

Com a construção dos novos reservatórios tem-se a possibilidade de aumentar o armazenamento de água na bacia do ribeirão São Bartolomeu de $66.596 \mathrm{~m}^{3}$ em caso de implementação do reservatório R2, ou de $375.216 \mathrm{~m}^{3}$, considerando o reservatório R1. Tais valores são apreciáveis tendo em vista a capacidade atual de armazenamento de água dos reservatórios localizados na UFV, que é de $143.270 \mathrm{~m}^{3}$. Também é importante ressaltar que na área dos barramentos não existe qualquer infraestrutura pública ou privada que possa ser atingida diretamente com a estrutura da barragem, conforme pode ser observado na imagem classificada (Figuras 3 e 4).

Em relação aos aspectos construtivos, ressalta-se que, a partir dos valores calculados de altura total (Tabela 7), é necessária a implementação de um barramento muito elevado para ambas as barragens, o que encarece o custo da obra, caso se almeje a exploração da máxima capacidade de regularização das bacias.

Como pode ser observado nas Tabelas 6 e 7, o reservatório denominado $\mathrm{R} 1$ apresenta uma maior área de drenagem e consequentemente maior capacidade de regularização da vazão, sendo esse o mais indicado do ponto de vista hidrológico, uma vez que o mesmo pode suprir a demanda hídrica da região por mais tempo. Em termos limnológicos, o R1 também é o mais recomendado devido a menores valores de sua profundidade relativa em comparação ao reservatório menor, o que potencializa a manutenção de uma melhor qualidade da água no reservatório. Porém, o impacto ambiental ocasionado pela emissão de gases do efeito estufa proveniente da degradação da matéria orgânica submergida (LI; ZHANG, 2014) é relativamente maior para a área do espelho d'água do R1, na qual é 5 vezes maior em comparação à área do R2.

\section{CONCLUSÕES}

Tendo em vista o quadro de escassez hídrica no município de Viçosa, frente ao elevado potencial de regularização de vazão na bacia do São Bartolomeu, recomenda-se a implantação de um dos reservatórios, com aumento da disponibilidade hídrica para fins de abastecimento público de $375.213 \mathrm{~m}^{3}$ e $66.596 \mathrm{~m}^{3}$ em caso de implementação do R1 e R2, respectivamente. Tal medida é apreciável tendo em vista a capacidade atual de armazenamento dos reservatórios localizados na UFV de $143.270 \mathrm{~m}^{3}$.

Entretanto, são necessários maiores estudos em relação à potencialidade de aumento dos conflitos por recursos hídricos na bacia do ribeirão São Bartolomeu, caso a regularização de vazão venha a ser implementada. Adicionalmente, ressalta-se a necessidade da avaliação de potenciais alterações na qualidade da água do rio a jusante dos reservatórios propostos.

Tendo em vista os valores extremamente elevados de profundidade relativa decorrente das características topográficas da região, é de suma importância o monitoramento constante da qualidade da água nos reservatórios propostos, visando a tomada de medidas de conservação do seu entorno que garantam a manutenção dos usos dos recursos hídricos por mais tempo. Ademais, recomenda-se a investigação de demais parâmetros limnológicos para melhor caracterização morfométrica e de transportes de nutrientes na massa líquida.

Além da construção de reservatórios visando o aumento da capacidade de regularização na bacia do ribeirão São Bartolomeu, são necessárias ações que visem a recuperação do potencial de recarga na bacia, destacadamente com foco em práticas conservacionistas que objetivam a proteção do solo e o uso e cobertura vegetal adequados na área de contribuição da bacia.

\section{AGRADECIMENTOS}

Os autores agradecem ao apoio financeiro da FAPEMIG, CAPES e CNPq no desenvolvimento deste trabalho.

\section{REFERÊNCIAS}

ALTHOFF, D.; RODRIGUES, L. N.; SILVA, D. D. da; BAZAME, H. C. Improving methods for estimating small reservoir evaporation in the Brazilian Savanna. Agricultural Water Management, Amsterdam, v. 216, p. 105-112, 2019. DOI: https://dx.doi.org/10.1016/j.agwat.2019.01.028

CARMO, E. J.; FERREIRA, I. O. Mapeamento dos relevos submersos das lagoas da UFV. Viçosa, MG: UFV, 2012. Relatório.

CHOW, V.T.; MAIDMENT, D.R.; MAYS, L.W. Applied hydrology. 1. ed. New York: McGraw-Hill, 1988. 572 p. 
EUCLYDES, H.P. Saneamento agrícola: Atenuação das cheias; metodologia e projeto. 2. ed. Belo Horizonte: RURALMINAS, 1988. 320 p.

GARCIA-CUERVA, L.; BERGLUND, E. Z.; BINDER, A. R. Public perceptions of water shortages, conservation behaviors, and support for water reuse in the U.S. Resources, Conservation and Recycling, v. 113, p. 106-115, out. 2016. DOI: https://dx.doi.org/10.1016/j.resconrec.2016.06.006

GIONGO, P. R.; VETTORAZZI, C. A. Albedo da superfície por meio de imagens TM-Landsat 5 e modelo numérico do terreno. Revista Brasileira de Engenharia Agrícola e Ambiental, Campina Grande, v. 18 , n. 8 , p. 833-838., 2014. DOI: http://dx.doi.org/10.1590/18071929/agriambi.v18n08p833-838

HOGEBOOM, R. J.; KNOOK L.; HOEKSTRA, A.Y. The blue water footprint of the world's artificial reservoirs for hydroelectricity, irrigation, residential and industrial water supply, flood protection, fishing and recreation. Advances in Water Resources, Amsterdam, v. 113, p. 285-294, mar. 2018.2 DOI: https://dx.doi.org/10.1016/j.advwatres.2018.01.028

HOLANDA, F. S. R.; ISMERIM, S. S.; ROCHA, I. P. DA; JESUS, A. S. DE; ARAÚJO FILHO, R. N. DE; MÉLLO JÚNIOR, A. V. DE. Environmental Perception of the São Francisco Riverine Population in Regards to Flood Impact. Journal of Human Ecology, New Delhi, v. 28, n. 1, p.37-46, 2009.

IBGE. IBGE cidades. Disponível em: $<$ https://cidades.ibge.gov.br/brasil/mg/vicosa/panora ma>. Acesso em: 30 set. 2019.

KOLADA, A. 2014. The effect of lake morphology on aquatic vegetation development and changes under the influence of eutrophication. Ecological Indicators, v. 38, p. 282-293. DOI: https://doi.org/10.1016/j.ecolind.2013.11.015

LEROUX, A. D.; MARTIN, V. L; ZHENG, H. Addressing water shortages by force of habit. Resource and Energy Economics, v.53, p.42-61, 2018. DOI: https://dx.doi.org/doi:10.1016/j.reseneeco.2018.02.004

LI, S.; ZHANG, Q. Carbon emission from global hydroelectric reservoirs revisited. Environmental Science and Pollution Research, v. 21, n. 23, p. 1363613641, 2014. DOI: https://dx.doi.org/10.1007/s11356014-3165-4

LOON, A. F. VAN; LANEN, H. A. J. VAN. Public perceptions of water shortages, conservation behaviors, and support for water reuse in the U.S. Resources, Conservation and Recycling, v. 113, p. 106-115, 2016. DOI:

https://dx.doi.org/10.1016/j.resconrec.2016.06.006

MARTINS, D. DE M. F.; CHAGAS, R. M.; MELO NETO, J. DE O. MÉLLO JÚNIOR, A. V. Impactos da construção da usina hidrelétrica de Sobradinho no regime de vazões no Baixo São Francisco. Revista Brasileira de Engenharia Agricola e Ambiental, Campina Grande, v. 15 , n. 9, p. 1054-1061, 2011. DOI: http://dx.doi.org/10.1590/S1415-43662011001000010

MATOS, A. T., SILVA, D. D., PRUSKI, F. F. Barragens de terra de pequeno porte. Viçosa: Editora UFV, 2012. $136 \mathrm{p}$.
MEKONNEN, M. M.; HOEKSTRA, A. Y. The blue water footprint of electricity from hydropower. Hydrol. Earth Syst. Sci., v. 16, n. 1, p. 179-187, 2012. DOI: https://dx.doi.org/10.5194/hess-16-179-2012

MORENO, Y. M.; VÉLEZ, F.; RAMÍREZ, N. A. Características morfométricas de un lago de plano inundable tropical (ciénaga Hoyo Los Bagres, Colombia). Revista Facultad de Ingeniería Universidad de Antioquia, n. 59, p. 203-214, jun. 2011.

NETO, J. F. B.; COELHO, R. M. P. A morfometria e o estado trófico de um reservatório urbano: Lagoa do Nado, Belo Horizonte, Estado de Minas Gerais. Acta Scientiarum - Biological and Health Sciences, Maringá, v. 24, n. 2, p. 285-290, 2002.

PAUL, N.; ELANGO, L. Predicting future water supplydemand gap with a new reservoir, desalination plant and waste water reuse by water evaluation and planning model for Chennai megacity, India. Groundwater for Sustainable Development, v. 7, p. 8-19, set. 2018. DOI: https://dx.doi.org/10.1016/j.gsd.2018.02.005

PEARCE, F. Dams make water shortages even worse. New Scientist, v. 234, n. 3131, p. 16, 2017. DOI: https://dx.doi.org/10.1016/S0262-4079(17)31201-0

PENMAN, H. L. Evaporation: an introductory survey. Netherlands Journal of Agricultural Science, Cambridge, v. 4, n. 1, p. 9-29, 1956.

PENMAN, H. L. Natural evaporation from open water, bare soil and grass. Proceedings of the Royal Society, London, v. 193, n 1032, p. 120-145, 1948. DOI: https://dx.doi.org/10.1098/rspa.1948.0037

PEREIRA, D. DOS R.; MARTINEZ, M. A.; PRUSKI, F. F.; SILVA, D. D. da. Hydrological simulation in a basin of typical tropical climate and soil using the SWAT model part I: Calibration and validation tests. Journal of Hydrology: Regional Studies, v. 7, p. 14-37, 2016. DOI: https://dx.doi.org/10.1016/j.ejrh.2016.05.002

PEREIRA, L. F.; CALEGARIO, A. T.; PEREIRA, S. B.; ARAÚJO, U. L.; SILVA, L N. O.; FERNANDES FILHO, E, I, Caracterização e mapeamento da degradação e intensidade de uso da terra exercida por pastagens. In: Simpósio Mineiro de Ciência do Solo, 4., Anais... Viçosa: [s.i.], p. 1-3, 2017.

PEREIRA, M. F. V. Contradições de uma "cidade científica": processo de urbanização e especialização territorial em Viçosa (MG). Caminhos de Geografia - revista on line, Uberlândia, v. 18, n. 16, p. 197-206, 2005.

RIPPL, W. Capacity of Storage Reservoirs for Water Supply. Minutes of Proceedings of the Institution of Civil Engineers, London, v. 71, p. 270-278,1883.

RODRIGUES, J. M.; AMORIM, M. C. DE; SEDIYAMA, G. C.; COSTA, L. C.; RODRIGUES, R. DE Á. DIAS, A. DA S. CONSUMO DE ÁGUA COMO ELEMENTO ESTIMADOR DE POPULAÇÃO URBANA. In: encontro de geógrafos de América Latina, 12., Anais... Montevideo: Observatorio Geográfico de América Latina, 14 p., 2009.

SCHÄFER, A. E.; MARCHETT, C. A.; SCHUH, S. M.; AHLERT, S.; LANZER, R. M. Morphological characterization of eighteen lakes of the north and middle coast of Rio Grande do Sul, Brazil. Acta Limnologica Brasiliensia, Rio Claro, v. 26, n. 2, p. 199-214, 2014. DOI: $\quad$ http://dx.doi.org/10.1590/S2179975X2014000200010 
SHAMSUZZOHAA, M; RASHEDUZZAMANA, M; GHOSH, R. C. Building Resilience for Drinking Water Shortages through Reverse Osmosis Technology in Coastal Areas of Bangladesh. Procedia Engineering, v. 212, p.559-566, 2018.2 DOI: https://dx.doi.org/10.1016/j.proeng.2018.01.072

TORRES, C. J. F.; BRAMBILLA, M.; FONTES, A. S.; MEDEIROS, Y. D. P. Conflitos pelo uso da água para a irrigação, geração de energia. Revista Gestão \& Sustentabilidade Ambiental, Palhoça n. esp., p. 195210 , 2015.

DOI:
ZHAO, D.; LIU, J. A new approach to assessing the water footprint of hydroelectric power based on allocation of water footprints among reservoir ecosystem services. Physics and Chemistry of the Earth, Oxford, v. 79-82, p. 40-46,

2015. https://dx.doi.org/10.1016/j.pce.2015.03.005

ZHUO, L.; HOEKSTRA, A. Y.; WU, P.; ZHAO, X. Monthly blue water footprint caps in a river basin to achieve sustainable water consumption: The role of reservoirs. Advances in Water Resources, Southampton, v. 113, p. 285-294, 2018. DOI: https://doi.org/10.1016/j.advwatres.2018.01.028 